\title{
CONTENT-DEPENDENT MULTIPURPOSE WATERMARKING RESISTANT AGAINST GENERALIZED COPY ATTACK
}

\author{
Chun-Shien Lu and Chao-Yong Hsu \\ Institute of Information Science, Academia Sinica, Taipei, Taiwan, ROC \\ e-mail: $\{$ lcs,cyhsu $\} @$ iis.sinica.edu.tw
}

\begin{abstract}
The attacks that can disclose or counterfeit the hidden information, are especially considered in this paper. We first explore the copy and collage attacks, and find that they can be specified as the generalized copy attack (GCA). Then, we propose to embed a single type of watermarks to achieve multiple purposes of content protection and authentication. Our scheme mainly relies on the deployment of contentdependent watermarks (CDWs), where each is a combination of an informative watermark and a robust hash. Mathematical analyses and experiment results consistently verify the effectiveness of the proposed scheme.
\end{abstract}

\section{INTRODUCTION}

To achieve robustness is still a challenging task in digital watermarking. Usually, removal and geometrical attacks inevitably alter the content/shape of media data in a blind manner. However, watermark-estimation attacks exist to defeat a watermarking system without certainly sacrificing media quality. In content protection, the collusion attack, which is a removal attack, and the copy attack, which is a protocol attack, are typical examples that can achieve the aforementioned goal. In content authentication, the counterfeit attack or collage attack is able to create the ambiguity problem. Usually, prior information is exploited by pirates to accomplish the above-mentioned attacks.

The conventional copy attack [4] is operated by estimating an invisible signal from one stego data and then copying it to another cover data. We call Kutter et al.'s copy attack, invisible-watermark-copy attack (IWCA). In addition, the collage attack [3] enforces to copy a partial and visible watermarked content from one stego data to another (combination of two genuine images). We call this type of counterfeit attacks, visible-content-copy attack (VCCA). Since both IWCA and VCCA involve the similar concept of "copy", we call them, generalized copy attack (GCA).

On the other hand, the collusion attack tries to collect and analyze a set of stego data so that unwatermarked copies can be constructed to create the false negative problem. The collusion attack naturally occurs in video watermarking.
However, we argue in $[6]^{1}$ that the collusion attack is not exclusively applied to video watermarking. On the contrary, it can also defeat those image watermarking methods that embed multiple redundant watermarks to resist geometrical distortions and locate malicious tampering.

Here, we treat the behaviors of VCCAs as malicious tampering so that its effects should be detected by fragile/semifragile watermarks. On the other hand, the embedded watermarks are expected to resist malicious attacks (including IWCA and collusion). Therefore, we will propose in this paper a new watermarking scheme, which can serve for content protection and authentication simultaneously while the powerful but easy to be executed attacks (GCA and collusion attack) can be resisted as well.

\section{MULTIPURPOSE WATERMARKING}

Almost all existing watermarking schemes are designed for either ownership protection or content authentication. In [5], we proposed a multipurpose watermarking scheme by hiding multipurpose watermarks at the same time. The validity of our method is based on switching the detection of the robust watermark and the fragile watermark. As a consequence, the order of hiding [7] is no longer an important issue.

Latterly, Deguillaume et al. also proposed a hybrid watermarking method [1] joining a robust watermark and a fragile watermark so that both copyright protection and content authentication can be achieved. In particular, they focus on the resistance to tampering and protocol attack (including copy and collage attacks). In the robust part of [1], they relies on a previously developed block-based multiple redundant watermark embedding technique [9]. With this unique characteristic, each image unit (e.g., blocks) can be treated in an image like a frame in a video; in this way, collusion attacks can be equally applied to those image watermarking methods that employ a multiple redundant watermark embedding strategy. As we analyzed and verified in [6], by means of collusion Voloshynovskiy et al.'s redundant watermarks were completely removed (as shown in Fig. 1)

\footnotetext{
${ }^{1}$ Watermark-estimation attacks (WEAs) were used to represent both the collusion and IWCA, which involve the similar estimation operation.
} 
before their excellent resistance to geometrical attacks can function. It is worth mentioning that the major difference of treating IWCA between this paper and [1] is that the copied invisible signal is regarded by us as meaningless (we use robust watermark to resist it) and by Deguillaume et al. as meaningful (they use fragile watermark to reveal it).

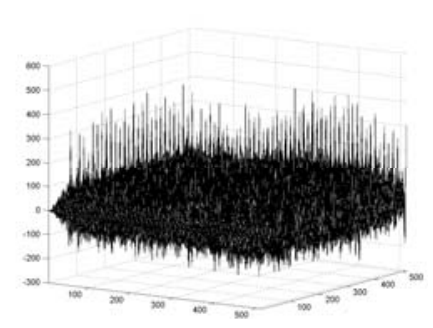

(a)

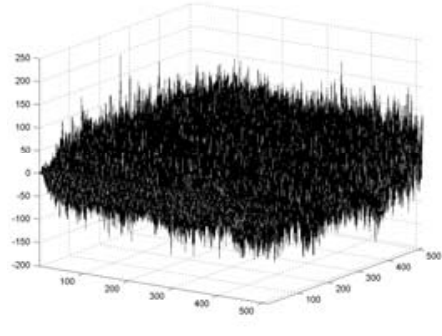

(b)
Fig. 1. Removal of Voloshynovskiy et al.'s [9] watermark peaks (3D inspection): peaks are clearly detected before collusion (a) but are mostly removed after collusion (b)[6].

\section{THE PROPOSED METHOD}

\subsection{Image Watermarking}

Our embedding procedure is carried out in a block-based manner. Various block relationship is depicted in Fig. 2. For each image $\mathbf{I}$, it will be divided into macroblocks of size $M \times 2 M$ (containing two $M \times M$ blocks). In a macroblock, a watermark sequence of 64 bits is embedded for both content protection and authentication. In this way, a periodical signal composed of many watermarks like the one [9] is concealed. In our scheme, a macroblock/block is the minimum unit, where content protection/authentication can be determined, respectively.

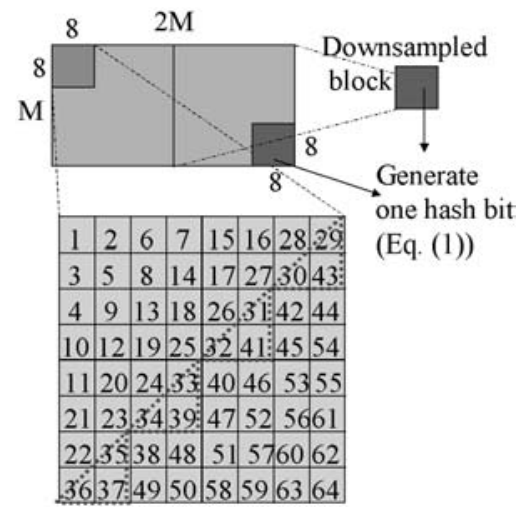

Fig. 2. Block relationship $(M=32)$ : one watermark bit is embedded in a pair of triangular areas in a block pair.

At the embedding stage, each $M \times M$ block is further divided into $8 \times 8$ blocks (denoted as $\left.B_{8}(\cdot)\right)$ and each $8 \times 8$ block pair is embedded with 4 watermark bits. It is said that a watermark bit is embedded if the following relationship between the AC energies of a block pair, $B_{8}(i)$ and $B_{8}(j)$, is satisfied: (i) if " 1 " is embedded, let $A C_{\text {eng }}\left(B_{8}(i)\right)>$ $A C_{\text {eng }}\left(B_{8}(j)\right)$; (ii) if " 0 " is embedded, let $A C_{\text {eng }}\left(B_{8}(i)\right)<$ $A C_{\text {eng }}\left(B_{8}(j)\right)$. The AC energy in a $8 \times 8$ block is defined to be the magnitude summation of the AC coefficients selected within a dashed triangular area, as shown in Fig. 2. Besides, the block pairs in an $M \times M$ block is determined by a secret key, which is the same in generating a watermark. At the detection stage, the hidden bit is extracted by checking the above relationship between a pair of blocks.

For copyright protection, all the watermarks extracted from $M \times 2 M$ blocks can be integrated to get the final but more accurate estimation. This operation is similar to collusion but is performed from an owner's viewpoint. As we have studied in [6], this is a very efficient step in accurately estimating the hidden watermark. For content authentication, the watermark detection result obtained from each $M \times M$ block is verified, as will be described in Sec. 3.3.

\subsection{Block Hash and Content-dependent Watermark}

In order to overcome GCA, we have to make the embedded watermarks dependent on its cover data. Meanwhile, the content-dependent information must be secured by a secret key and be robust to digital processing in order not to damage watermark detection. To this end, we introduce image hash and incorporate it with the watermark to create the so-called content-dependent watermark (CDW).

Here, the proposed hash extraction procedure is operated in the $8 \times 8$ block-DCT domain. In an $M \times M$ block (denoted as $B_{M}(\cdot)$ ), a random sequence of $8 \times 8$ blocks is first generated. Besides, for each $M \times M$ block $B_{M}(m)$ it is also downsampled to be an $8 \times 8$ block, denoted as $B_{8}^{d s}(m)$. For each block pair, $B_{8}(i)$ and $B_{8}^{d s}(m)$, a piece of representative but robust information is created. It is defined as the magnitude relationship between two DC coefficients:

$M H(k)= \begin{cases}+1, & \text { if }\left|D C\left(B_{8}(i)\right)\right|-\left|D C\left(B_{8}^{d s}(m)\right)\right| \geq 0 \\ -1, & \text { otherwise, }\end{cases}$

where $M H(k)$ is an element of a hash sequence $\mathbf{M H}$ and $k=16 \times m+i, 1 \leq i \leq 16$. As we will describe in Sec. 3.3 , the downsampled block is extremely helpful in revealing the boundary of images that are collaged together. We call this feature value $M H(\cdot)$ robust because this magnitude relationship can be mostly preserved under incidental modifications (e.g., compressions, filtering, and denoising).

In the aspect of content-dependent watermark generation, the media mash $\mathbf{M H}_{m}$ of an $M \times M$ block $B_{M}(m)$ is mixed with a bipolar watermark, $\mathbf{W}$, to generate a contentdependent watermark $\left(\mathbf{C D W} \mathbf{W}_{m}\right)$ as

$$
\mathbf{C D W}_{m}=S\left(\mathbf{W}, \mathbf{M H}_{m}\right),
$$


where $S(\cdot, \cdot)$ is a mixing function and will be designed as a procedure of permuting the hash $\mathbf{M H}_{m}$ using the same secret key $K$, followed by shuffling the watermark to enhance security. The sequence $\mathbf{C D W}_{m}$ is what we will embed into $m$-th $M \times M$ block, $B_{M}(m)$.

\subsection{Resistance to Generalized Copy Attack (GCA)}

\subsubsection{Resistance to Invisible-Watermark-Copy Attack}

Let $\mathbf{M H}_{\mathbf{X}}$ and $\mathbf{M H}_{\mathbf{Z}}$ denote the hash sequences generated from two different $M \times M$ blocks, $\mathbf{X}$ and $\mathbf{Z}$, respectively. In addition, let $\mathbf{C D W}_{\mathbf{X}}$ denote the content-dependent watermark to be hidden into $\mathbf{X}$ and let the watermark estimated from $\mathbf{X}^{\mathbf{s}}$ be $\mathbf{W}^{\mathbf{x}}$, which will contain partial information from $\mathbf{C D W}_{\mathbf{X}}$. By directing the copy attack at the target block $\mathbf{Z}$, we can get the counterfeit watermarked block $\mathbf{Z}^{\mathbf{s}}$. Later, in the detection process, the content-dependent watermark, $\mathbf{W}^{\mathbf{z}}$, estimated from block $\mathbf{Z}^{\mathbf{s}}$ will be

$$
\mathbf{W}^{\mathbf{z}}=\operatorname{sgn}\left(\alpha \times \mathbf{C D W}_{\mathbf{X}}+\mathbf{n}\right) \approx \mathbf{C D W}_{\mathbf{X}},
$$

where $\mathbf{n}$ is a noise sequence and $\operatorname{sgn}(\cdot)$ determines the sign of its argument. Given Eqs. (2) and (3), the bit error rate (BER) calculated from $\mathbf{C D W}_{\mathbf{Z}}$ and $\mathbf{W}^{\mathbf{z}}$ can be derived to show resistance to copy attack based on blocks $\mathbf{X}$ and $\mathbf{Z}$ that are dissimilar:

$$
\begin{aligned}
\operatorname{BER}\left(\mathbf{C D W}_{\mathbf{z}}, \mathbf{W}^{\mathbf{z}}\right) & \approx \operatorname{BER}\left(\mathbf{C D W}_{\mathbf{z}}, \mathbf{C D W}_{\mathbf{X}}\right) \\
& \approx 0.5 .
\end{aligned}
$$

\subsubsection{Resistance to Visible-Content-Copy Attack}

Let $\mathbf{W}^{\mathbf{e}}{ }_{m}$ be a watermark estimated from the $m$-th block after eliminating the incorporated image hash. According to how the image hash $\mathbf{M H}_{m}$ is generated, we have

$\operatorname{BER}\left(\mathbf{W}_{m}^{\mathbf{e}}, \mathbf{W}\right)\left\{\begin{array}{l}=\epsilon, \text { if } \mathbf{M} \mathbf{H}_{m} \text { is from the same image, } \\ =\zeta>>\epsilon, \text { otherwise, }\end{array}\right.$

where $\epsilon$ ideally approximates 0 and $\zeta$ depends on how different image sources constitute an image block, as shown in Fig. 3. When an image has been incidentally modified, $\epsilon$ may (slightly) be increased. The degree of increase depends on the resistance of a watermarking scheme to incidental modifications. Based on Eq. (5), we propose to use binary thresholding to classify incidental modifications and malicious distortions. Statistically, this threshold $T$ can be adaptively determined as $\mu+q \times \rho$ based on the assumptions that (i) the larger distribution of BERs form a Gaussian distribution with mean $\mu$ and variance $\rho^{2}$ (basically, it comes from a genuine image with/without incidental modifications, as shown in Fig. 4.); (ii) the false negative probability $Q(q)$ $(Q(\cdot)$ is referred to as a Gaussian Integral Function) of deciding an image block that is credible as has been maliciously tampered with is small.

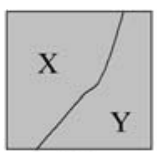

(a)

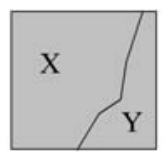

(b)

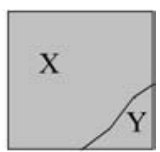

(c)
Fig. 3. Different scenarios of collaged blocks generated from two different image sources (X and Y). For (a), $\zeta$ (in Eq. (5)) would approximate 0.5.

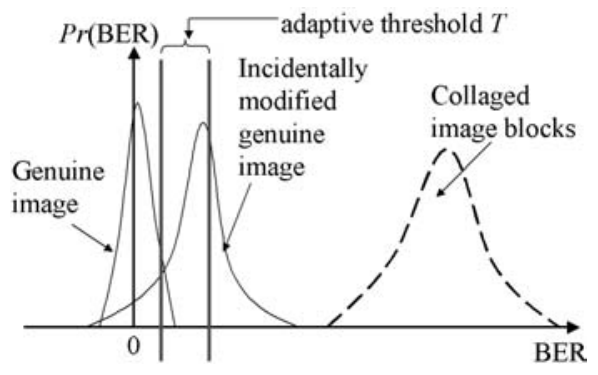

Fig. 4. Different scenarios of distributions of BERs.

\section{EXPERIMENTAL RESULTS}

The resistance of our scheme to GCA will be demonstrated but the results of resisting collusion and other attacks are not shown here due to limits of space.

\subsection{CDW Resistance to IWCA}

Ten varieties of gray-scale cover images of size $512 \times 512$ were used. One of them was watermarked, estimated, and copied to the other 9 unwatermarked images to form 9 counterfeit stego images. By repeating this procedure, 90 counterfeit stego images were obtained. All BERs obtained by applying IWCA to our method fell within [0.4847 0.5198] (all were sufficiently larger than $T$ ), which indicates the absence of watermarks. Obviously, the experimental results are consistent with the analytic result (Eq. (4)).

\subsection{CDW Resistance to VCCA}

One typical result, as shown in Fig. 5, was chosen to demonstrate the effectiveness of our method. The left portion of Fig. 5(a) was copied and pasted into Fig. 5(b) to form a collaged image (Fig. 5(c)). By employing our scheme, Fig. 5(d) showed the block boundaries detected between two stego images. In addition, our result revealed that block boundaries are hard to be detected if image hashing is not used. This clearly explains the benefit of employing contentdependent hashing in resisting collage attacks. On the other hand, Fig. 5(c) was further compressed with JPEG quality factors $90 \%$ and $80 \%$ to test the robustness and fragility. Figs. 5(e) and (f), respectively, illustrate that the collaged 
boundaries are located quite precisely while the JPEG effects begin to be slightly detected when $Q F$ increases to $80 \%$. To deal with resistance to compression with lower quality factors, we propose to combine our scheme with a technique [2] that can determine whether an image has been JPEG compressed.

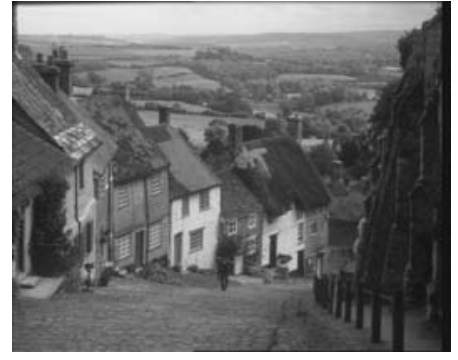

(a) stego goldhill

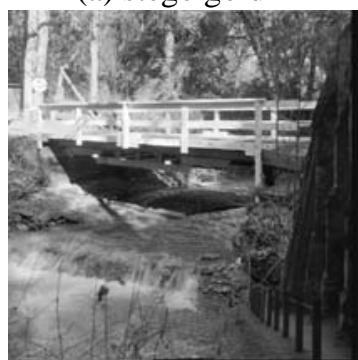

(c) collaged image

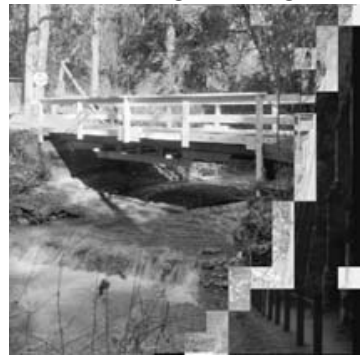

(e)(c)+JPEG QF90\%

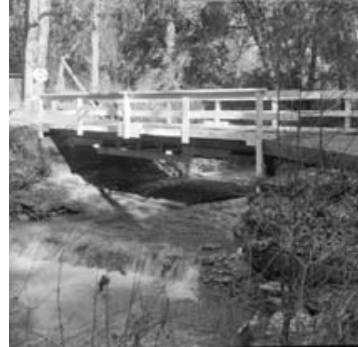

(b) stego bridge

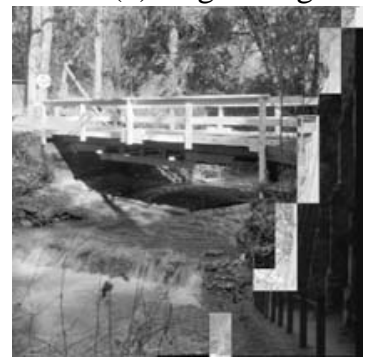

(d)tampering detection of (c)

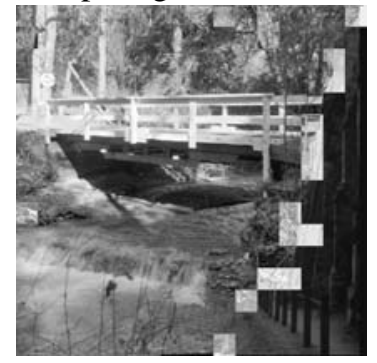

(f)(c)+JPEG QF80\%
Fig. 5. Content-dependent watermark resistant to WCCA.

\subsection{Remarks and Comparisons}

After describing the proposed method and demonstrating the experimental results, we acquire some remarks: (i) Since the motivation of this paper is to propose a watermarking scheme for multiple applications, the design principle will be restricted to embed a watermark signal into a local region. Compared with global watermarking that only a watermark is embedded into a whole cover data, the achievable robustness of local watermarking (e.g., the one presented here) has been reduced. (ii) The introduced image hash is extremely helpful in resisting copy and collage attacks. Compared with existing methods, the collage attack cannot be tolerated in [8] and the authentication part of [1] is purely fragile. (iii)
Instead of concealing a noise-like watermark (e.g. [9]), a certain relationship of DCT block-pair's middle frequency energy is enforced to embed a watermark bit in this paper. Based on (iii), no prior knowledge can be exploited by the collusion attack to remove the hidden signal while improving the media's quality. (iv) The most challenging problem for multipurpose watermarking is how to resist geometrical distortions. No solution in the literature was found.

\section{CONCLUSIONS}

Although multiple block-based watermarks can be embedded to withstand geometrical distortions and reveal malicious tampering, they are vulnerable to be colluded, copied, and collaged such that the desired functionalities are lost. To cope with these attacks simultaneously, a novel multipurpose watermarking scheme has been investigated in this paper based on the idea of content-dependent watermark. To our knowledge, the proposed scheme is the first to enable both resistance to the collusion and the generalized copy attack and to serve for multiple applications, simultaneously. The addressed remarks are worth researching in the future.

\section{REFERENCES}

[1] F. Deguillaume, S. Voloshynovskiy, and T. Pun, "Secure Hybrid Robust Watermarking Resistant against Tampering and Copy Attack", Signal Processing, Vol. 83, 2003.

[2] Z. Fan and L. Queiroz, "Identification of Bitmap Compression History: JPEG Detection and Quantizer Estimation," IEEE Trans. on Image Processing, Vol. 12, pp. 230-235, 2003.

[3] M. Holliman and N. Memon, "Counterfeiting Attacks on Oblivious Block-wise Independent Invisible Watermarking Schemes”, IEEE Trans. on Image Processing, Vol. 9, No. 3, pp. 432-441, 2000.

[4] M. Kutter, S. Voloshynovskiy, and A. Herrigel, "The Watermark Copy Attack", Proc. SPIE: Security and Watermarking of Multimedia Contents II, Vol. 3971, 2000.

[5] C. S. Lu and H. Y. Mark Liao, "Multipurpose Watermarking for Image Authentication and Protection", IEEE Trans. on Image Processing, Vol. 10, No. 10, pp. 1579-1592, 2001.

[6] C. S. Lu and C. Y. Hsu, "Content-dependent Anti-Disclosure Image Watermark", Proc. Int. Workshop on Digital Watermarking, LNCS 2939, Seoul, Korea, 2003.

[7] F. Mintzer and G. W. Braudaway, "If one watermark is good, are more better?", Proc. IEEE Int. Conf. on Acoustic, Speech, and Signal Processing, pp. 2067-2070, 1999.

[8] A. H. Paquet, R. K. Ward, and I. Pitas, "Wavelet Packets-based Digital Watermarking for Image Verification and Authentication,” Signal Processing, Vol. 83, pp. 2117 2132, 2003.

[9] S. Voloshynovskiy, F. Deguillaume, and T. Pun, "Multibit Digital Watermarking Robust against Local Nonlinear Geometrical Distortions", Proc. IEEE ICIP, pp. 999-1002, 2001. 